\title{
The GLE on Oct. 28, 2003 - radio diagnostics of relativistic electron and proton injection
}

\author{
H. Aurass, G. Mann, G. Rausche, and A. Warmuth
}

\author{
Astrophysical Institute Potsdam, 14482 Potsdam, Germany \\ e-mail: haurass@aip.de
}

Received 20 March 2006 / Accepted 10 June 2006

\begin{abstract}
Timing discrepancies between signatures of accelerated particles at the sun and the arrival times of the particles at near-earth detectors are a matter of fundamental interest for space-weather applications. The solar injection times of various components of energetic particles were derived by Klassen et al. (2005, JGR, 110, A09S04) for the October 28, 2003, X-class/ $\gamma$-ray flare in NOAA AR 10486. This flare occured in connection with a fast halo coronal mass ejection and a neutron monitor-observed ground level event (GLE). We used radio (Astrophysikalisches Institut Potsdam, WIND, Nançay Multifrequency Radio Heliograph), $\mathrm{H} \alpha$ (Observatorium Kanzelhöhe), RHESSI, SOHO (EIT, LASCO, MDI), and TRACE data to study the associated chromospheric and low coronal phenomena. We identify three source sites of accelerated particles in this event. Firstly, there is a source in projection $0.3 R_{\odot}$ away from AR 10486 , which is the site of the reconnection outflow termination, as revealed by a termination shock signature in the dynamic radio spectrum. Secondly, there is the extended current sheet above a giant coronal postflare loop system in the main flare phase. Thirdly, there is a source situated on a magnetic separatrix surface between several magnetic arcades and neighbouring active regions. This source is $0.2 R_{\odot}$ away from AR 10486 and acts during onset and growth of high energy proton injection in space. It is not clear if this source is related to the acceleration of protons, or if it only confirms that energetic particles penetrate a multistructure magnetic loop system after being previously accelerated near the main HXR-and $\gamma$-ray sources. The result is in favour of energetic particle acceleration in the low corona $\left(<0.5 R_{\odot}\right.$ above the photosphere) and in contrast to acceleration of the relativistic particles at remotely propagating shock waves.
\end{abstract}

Key words. acceleration of particles - Sun: radio radiation - Sun: magnetic fields - Sun: flares - Sun: coronal mass ejections (CMEs) - Sun: X-rays, gamma rays

\section{Introduction}

During the formation of a coronal mass ejection (CME), a part of the inner corona becomes unstable and is released into the outer corona and the heliosphere. This leads to a transient state of opening up and reclosure of the existing quasi-equilibrium plasma-magnetic field configuration. Depending on not yet clarified conditions, this process can be coupled with a flare in one or several active regions situated near the magnetic roots of the CME. During the flare, both impulsive and gradual energy release is observed. Due to well-known observational problems, the relative timing between the CME motion and the impulsive flare phase is ambiguous. There is also the suggestion that flares can be a (small-scale) consequence of a CME (Hiei et al. 1993; Hundhausen 1998).

Magnetic reconnection is one model for the coronal relaxation behind a CME and for flare energy release (e.g., Priest \& Forbes 2000). Particle acceleration during this process is not yet fully understood despite several indirect and direct diagnostics: nonthermal radio and hard X-ray emission of electrons, all signs of trapped and precipitated particles in the solar atmosphere, and in-situ observations of particle streams in space.

Considering the sources of accelerated particles, both CMEs and flares come into play. This is mainly due to the roughly $10 \mathrm{~min}$ average time delay reported between remote solar signatures of particle injection and back-extrapolated solar injection times of particle streams near earth (Krucker et al. 1999;
Haggerty \& Roelof 2002; Classen et al. 2003; Klein et al. 2005). Three sources of the accelerated particles measured near earth are invoked: the fast impulsive energy release during flare onset, a coronal shock wave ${ }^{1}$ and/or a shock wave driven ahead of the CME in the upper corona and in the heliosphere ${ }^{2}$, and the posteruptive energy release ${ }^{3}$. Note: with respect to the nonthermal electrons leading to radio emission, the coronal and interplanetary shocks are low-energy electron accelerators. In the given context, we have in mind near-relativistic electron and proton streams.

The X-class flare of October 28, 2003, is a well-observed event. Gros et al. (2004), Share et al. (2004), Miroshnishenko et al. (2005), Bieber et al. (2005), Simnett (2005), and Kiener et al. (2006) discussed hard X-ray, $\gamma$-ray, and particle data of this most intense solar energetic particle event of cycle 23 (Gopalswamy et al. 2005), which was also observed by neutron monitors on the ground. Pick et al. (2005) studied radio, $\mathrm{H} \alpha$, and white light observations and found a large spatial extent of the event. They come to the conclusion that the radio emission is ascribed to the CME-edge-reconfiguration of the coronal magnetic field. Klassen et al. (2005) find two substantially different

\footnotetext{
Remotely seen as a meter wave radio type II burst, Nelson \& Melrose (1985).

2 The Hm-km wave interplanetary radio type II burst.

3 During the relaxation of the disturbed corona, e.g. Cliver et al. (1986), and Klassen et al. (2005).
} 
Table 1. October 28, 2003 - particle stream timing based on Klassen et al. (2005).

\begin{tabular}{lll}
\hline \hline 28 October 2003 & Solar release time & Arrival at 1 AU (UT) \\
& & $11: 01: 20(<800 \mathrm{MHz}$, AIP $)$ \\
First radio signature & $10: 50$ & $10: 58(>800 \mathrm{MHz}$, AOO $)$ \\
& & $11: 21-11: 26$ \\
Impulsive electrons & $11: 05(11: 00-11: 09)$ & $11: 13-11: 19$ \\
Relativistic protons & $11: 07(11: 04-11: 10)$ & $11: 25-11: 36$ \\
Gradual electrons & $11: 19(11: 14-11: 25)$ & 1 \\
\hline
\end{tabular}

Explanation: in brackets: uncertainties of back-extrapolation. The interval in $1 \mathrm{AU}$ times: different detector channels. AOO data: courtesy M. Karlický.

Table 2. October 28, 2003 - synopsis.

\begin{tabular}{|c|c|c|}
\hline Effect & Range, instrument & $\begin{array}{l}\text { Description } \\
\text { (all times in UT, all speeds in } \mathrm{km} \mathrm{s}^{-1} \text { ) }\end{array}$ \\
\hline & LASCO-C2 & $\operatorname{CME} 7: 31 \operatorname{ESE} 114^{\circ} v=394, w=16^{\circ}$ \\
\hline $\begin{array}{l}\text { PRE- } \\
\text { FLARE }\end{array}$ & $\begin{array}{l}\text { GOES } \\
\text { Radio } \\
\text { LASCO-C2 } \\
\text { GOES } \\
\text { H } \alpha \\
\text { EIT, LASCO-C2 } \\
\text { H } \alpha \\
\text { H } \alpha \text { blue wing } \\
\text { RHESSI } \\
\text { Radio } \\
\text { LASCO-C2, EIT } \\
\text { EIT }\end{array}$ & $\begin{array}{l}\text { 8:50-10:58 } \\
\text { IIIGG 8:53-9:00 } \\
\text { CME 9:30 E } 88^{\circ} v=853, w=22^{\circ} \\
\text { soft X-ray flux time deriv. max. 9:55, 10:06, 10:29 } \\
\text { 9:43 small compact flaring, 10:03-10:09 } \\
\text { first kernels, motions at W-end of AR10486 filament } \\
\text { sigmoid 9:48-10:24, loop system in SW 10:54 } \\
\text { 10:22-10:40 big flare in progress } \\
\text { W-part 10486 AR-filament visible, expansion to SW } \\
\text { HXR source } 10: 33 \text { near E-end of sigmoid } \\
\text { spikes 10:23-26; } 10: 33-34, \text { cont. untill 10:40, some type III } \\
\text { CME SE } 124^{\circ}, 10: 54, v=1054, w=147^{\circ} \\
\text { 10:48-11:00 repeated sigmoid formation, W-wing less visible }\end{array}$ \\
\hline $\begin{array}{l}\text { IMPULSIVE } \\
\text { PHASE }\end{array}$ & $\begin{array}{l}\text { INTEGRAL } \\
\mathrm{H} \alpha \\
\text { TRACE } 284 \AA \\
\text { Radio } \\
\text { LASCO-C2 } \\
\text { RHESSI } \\
\mathrm{H} \alpha\end{array}$ & $\begin{array}{l}\text { 10:58-11:10 } \\
\text { dm start 10:58, m start 11:01 pulsation, narrowband line } \\
\text { short flash of a new source at AR10491 11:01:40-11:02:40 } \\
\text { TS F-H mode } 11: 02: 30-11: 04 \text {, source } 0.3 R_{\odot} \text { SW of AR } 10486 \\
\gamma \text {-ray pulse } \geq 10 \mathrm{MeV} 11: 02: 30-11: 03: 30(\mathrm{G} 04) \\
\text { AR-filament eruption between F2 and F3 (not directly observed) } \\
\text { 11:02:31-47 lift-off of bright matter behind X-threads } \\
\text { 1080 proj. speed } \\
\text { negative frequency drift kink in the TS spectrum at } 11: 03: 10 \\
(v \approx 2600) \text {, expansion on disc with CME speed }(v \approx 2300)(\mathrm{P} 05) \\
\text { Halo CME with SW looplike feature } 11: 30\left(w=79^{\circ}\right), v=2459 \\
\text { looptop \& footpoint sources in AR 10486, obs. start } 11: 06: 45 \\
\text { F6 changing length (visibility?) after imp. phase }\end{array}$ \\
\hline $\begin{array}{l}\text { MAIN } \\
\text { PHASE }\end{array}$ & $\begin{array}{l}\text { LASCO-C2 } \\
\mathrm{H} \alpha \\
\text { Radio } \\
\mathrm{H} \alpha \\
\text { SOHO,WIND }\end{array}$ & $\begin{array}{l}\text { past } 11: 10 \text { decay, long duration event } \\
11: 12-11: 22 \text { continuum } 1200-200 \mathrm{MHz} \text {, fiber burst patches } \\
\text { onset to max. of protons }(\geq 1.4 \mathrm{GeV}) \text { at } 1 \mathrm{AU} 11: 13-19 \text { (K05) } \\
\text { type II features } v \approx 3200 \\
11: 22 \text { closure of the corona (end of III/II-like effects at } 14 \mathrm{MHz}) \\
11: 25 \text { UT onset cont. with neg. freq. drift } v=30 \text { \& minute puls. } \\
11: 58-12: 05 \text { fast drift bursts with neg. drifting low-freq. cutoff } \\
\text { rising cusp with } v=1083 \text { toward SW between } 11: 30 \text { and } 11: 54 \\
\text { F5 } \approx 11: 44 \text { activation, motion toward F6; F7 11:49 activation } \\
3 \text { dm rise/falls in } 11: 40-12: 10 \\
11: 50-12: 10 \text { reactiv. of TS source, unspecific spectrum } \\
\text { activation F1 } \leq 12: 03: 48, \text { eruption } 12: 20: 40 \\
\text { onset of gradual electrons }(\geq 10.4 \mathrm{MeV}) \text { at } 1 \text { AU } 11: 25-11: 36 \\
\text { max. } \approx 16 \mathrm{~h}(\mathrm{~K} 05)\end{array}$ \\
\hline
\end{tabular}

Abbreviations: P05: Pick et al. (2005); K05: Klassen et al. (2005); G04: Gros et al. (2004).

electron ensembles for this event: an impulsive, softer component, and a gradual one with a harder electron energy spectrum. For both components, and for the ground level event (GLE)inducing stream of relativistic protons, a solar injection time was calculated from arrival times at near-earth detectors. For convenience, Table 1 repeats the particle data from Klassen et al. (2005).

In this paper we present our analysis of that event, and we compare the injection time results of Klassen et al. (2005) with our findings. They are mainly derived from the fine structure content of the radio burst spectrum ${ }^{4}$. In studying the radio data, we use a recently published method to identify flare-activated structures in the extrapolated coronal magnetic field by tracing fiber or intermediate drift burst sources (Aurass et al. 2005). Our results reveal that the relativistic electrons seen early in the

\footnotetext{
${ }^{4}$ We cannot clarify possible discrepancies between Klassen et al. (2005) and Bieber et al. (2005), which are related to the analysis of particle data and rely on the times in Table 1.
} 
event (Gros et al. 2004) are accelerated during the occurence of a strong termination outflow shock (TS) signature in the impulsive phase radio spectrum. This is type II burst-like radio emission of the standing fast mode shock wave formed at the termination of a hot reconnection outflow jet (Aurass et al. 2002; Aurass \& Mann 2004). Here, it is quite possible to associate the TS source site with other features describing the evolution of the flare and the CME. Further, we determine those part of the corona where we find - during the proton injection time given in Table 1 independent radio evidence for the presence of protons in the lower (post-CME) corona. We argue that the gradual source of energetic electrons (the third particle source discriminated by Klassen et al. 2005) is related to the main phase evolution of the flare current sheet on the background of the halo CME-disturbed corona.

\section{Instruments and tools}

Low-energy (1-10 keV) nonthermal electrons are the source of solar radio radiation due to the plasma emission mechanism (Melrose 1985). This electron population is studied using radio spectral data, which are mainly provided by the Astrophysikalisches Institut Potsdam (AIP) Radio Spectrograph, covering the range $40-800 \mathrm{MHz}$ with a time resolution of $0.1 \mathrm{~s}$ (Mann et al. 1992). This is supplemented by information about decimeter and microwave spectra $(0.8-4.5 \mathrm{GHz})$ from the Astronomical Institute Ondřejov (AOO, courtesy M. Karlický). The Dekameter/Hektometer regime is covered by the Radio Receiver RAD1 (1.075-13.825 MHz) of the Radio and Plasma Waves Instrument (WAVES) aboard the WIND spacecraft (Bougeret et al. 1995; and /lep694.gsfc.nasa.gov/waves/waves.html, courtesy M. Kaiser).

The positions of radio sources are obtained from the Nançay Multifrequency Radio Heliograph (NRH; Kerdraon \& Delouis 1997; courtesy A. Kerdraon \& K.-L. Klein), which provides radio heliograms at a cadence of 8 images/second at 6 frequencies between 450 and $150 \mathrm{MHz}$, with a beam size of $\approx 1$ arcmin at $327 \mathrm{MHz}$. In this paper we use only the NRH data at 432, $410.5,327$, and $236.6 \mathrm{MHz}$. In all figures only the source centroids assuming Gaussian source profiles or single-source flux curves are shown.

Whole-sun soft X-ray fluxes are recorded by the GOES satellites. They are used to characterise the time behaviour of the analysed flares and CMEs. Electrons from a few tens of keV up to many $\mathrm{MeV}$ produce hard X-ray (HXR) and $\gamma$-ray radiation due to thick-target bremsstrahlung. Spectral (from $3 \mathrm{keV}$ to $18 \mathrm{MeV}$ ) as well as imaging observations in this range are available from the Reuven Ramaty High Energy Solar Spectroscopic Imager (RHESSI; Lin et al. 2002; courtesy, the RHESSI Team, PI: R. P. Lin).

The Solar and Heliospheric Observatory (SOHO) is a project of international cooperation between ESA and NASA. Coronal structures are studied using full-disk Fe XII $195 \AA$ ( $\approx 1.5 \mathrm{MK})$ images from SOHO/EIT (Extreme Ultraviolet Imaging Telescope, Delaboudinière et al. 1995, and /umbra.nascom. nasa.gov/eit/eit_guide/, courtesy J.B. Gurman and the EIT Consortium). As another key to understanding coronal structures, we used Transition Region and Coronal Explorer (TRACE) images in Fe XII $195 \AA$, as well as in FeXV284 $(\approx 2 \mathrm{MK})$ (Handy et al. 1999; and /trace. Imsal. com/trace_cat.html, courtesy S.L. Freeland $\&$ A. Title). This is supplemented by full-disk $\mathrm{H} \alpha$ filtergrams (line centre and both wings) provided by the upgraded Patrol Telescope at Kanzelhöhe Solar Observatory (KSO, Otruba 1999; Otruba \& Pötzi 2003). The upgrade was a joint project of KSO and AIP.

The associated CME is studied using $\mathrm{SOHO/LASCO}$ (Large Angle Spectro-Coronograph, Brueckner et al. 1995), and the CME catalog at /cdaw.gsfc.nasa.gov/CME_list/ index.html, which is generated and maintained at the CDAW Data Center by NASA and The Catholic University of America, in cooperation with the Naval Research Laboratory, courtesy S. Yashiro \& N. Gopalswamy.

SOHO/MDI (Michelson Doppler Imager, Scherrer et al. 1995, and /soi.stanford.edu/magnetic/index5. html, courtesy the MDI Consortium) longitudinal field magnetograms are used as a photospheric boundary condition for potential extrapolations of the coronal magnetic field in Cartesian coordinates (Seehafer 1978; and courtesy A. Hofmann). Further, we took advantage of the PFSS branch in the Solar Software to access the potential field extrapolation for the whole sun in spherical coordinates (Schrijver \& DeRosa 2003; and courtesy M. L. DeRosa).

\section{Observations}

\subsection{Overview}

The event of interest (EOI) is located in the NOAA active region (AR) 10486 (S16, E08). This is the most extended and complex active region in a cluster of six different spot groups (Fig. 1a). The Cartesian potential field extrapolation shows that AR 10486 is magnetically connected with the surrounding active regions in a complex manner. At low heights (Fig. 1b, 35-70 Mm), the flaring region is situated in the SE of a circular arcade centred in the leading spots of AR 10486 . This structure is tangentially surrounded by three elongated arcades. KSO $\mathrm{H} \alpha$ data show that the NE part of the circular arcade carries a filament (F3 in Fig. 7, Sect. 3.3) which remains stable during the whole event.

At medium heights (Fig. 1c, 70-150 Mm), the field line pattern points to the presence of an almost closed separatrix surface between the central circular arcade and the surrounding field structures. This means that above the circular arcade, the top of an almost closed cover of low magnetic field strengths where several distinct field systems contact each other is situated. Démoulin et al. (1997) studied separatrix surfaces in observed magnetic configurations and their association with coronal energy release sites.

At a height of 150-300 Mm (Fig. 1d), some far-reaching connections become visible: there is a bridge from 10486 to 10488 and 10493 in the north across the circular arcade. Further, there are many field lines connecting ARs 10488 and 10489 . In AR 10488 , the far-reaching connections are mainly rooted in the trailing polarity. Notice that the large-scale field structure leaves a gap toward SW with almost no magnetic flux above $\approx 150 \mathrm{Mm}$ open (arrow in Fig. 1d). The erupting filament evolves into this gap during the flare. The open field lines visible in the W-part of Fig. 1d mainly reflect the box boundary and are not physically relevant.

We note that a similar nested magnetic configuration was discussed for another $\gamma$-ray flare (Aurass et al. 1998). In the following, we will see that the given short discussion of the coronal potential field structure of the complex of ARs already serves as a veritable guide through the wealth of available data. Table 2 gives a synopsis of the different observational facts and the data sources for the EOI. 


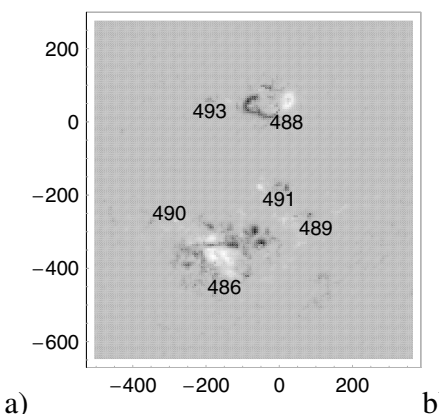

a)

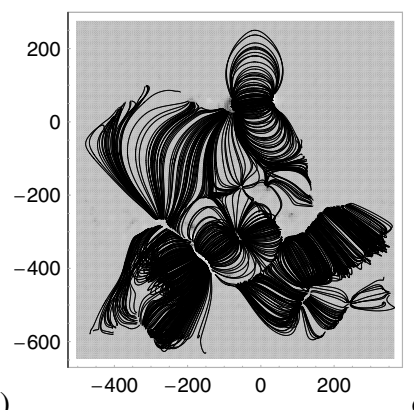

b)

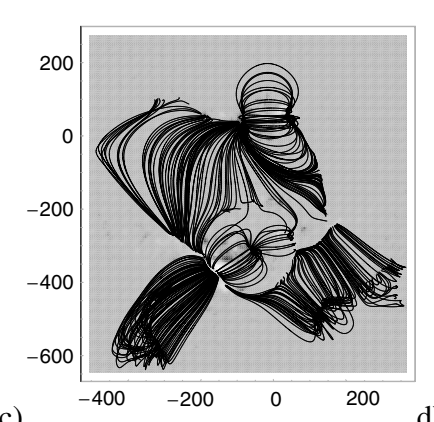

c)

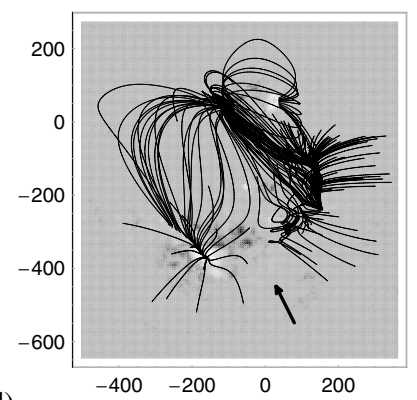

d)

Fig. 1. Panel a) SOHO-MDI 11:11 UT magnetogram, numbers give NOAA active region 10xxx. Coordinates: arcsec on the disc. Panels b)-d) potential field extrapolation, Cartesian coordinates (courtesy: A. Hofmann). Field lines turning in 36.5-73 Mm, 73-146 Mm, and >146 Mm or leaving the box, respectively. Main flare activity occurs in AR 10486. The arrow in d) points to a range of very low magnetic flux above $150 \mathrm{Mm}$ where the erupting filament evolves during the flare.

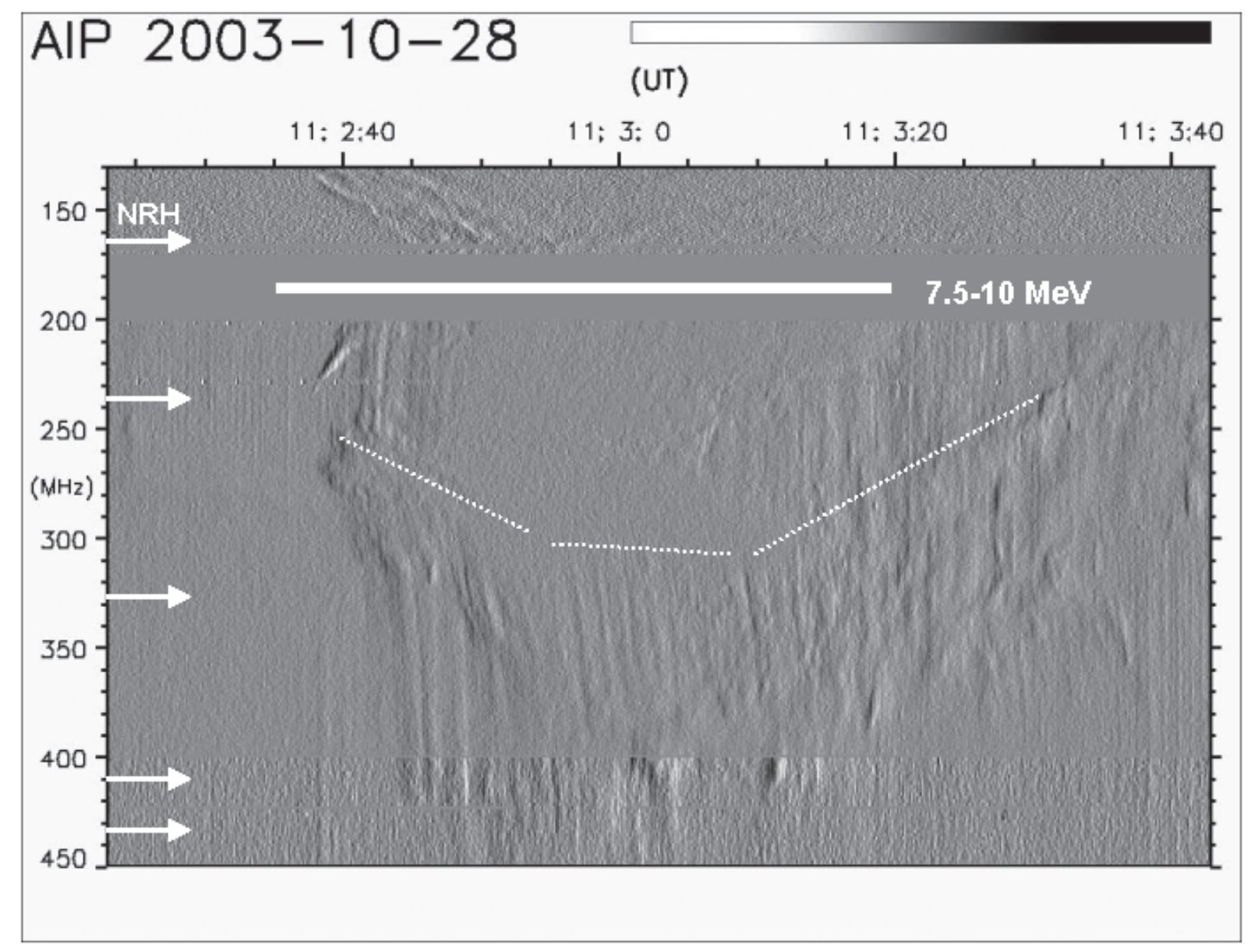

Fig. 2. The time-derived dynamic radio spectrum (AIP) of the Oct. 28, 2003, termination shock (TS). At $400 \mathrm{MHz}$, there is a transition between two receivers/aerials; $170-200 \mathrm{MHz}$ is a local observation gap. Arrows are inserted at the observing frequencies of the NRH, as is a thick white bar as the time interval of $\gamma$-ray emission (Gros et al. 2004). A small dashed white line guides the eye with respect to frequency drift tendencies.

We intend to contribute a refined analysis of two specific phenomena.

- A termination shock (TS) radio spectral signature during the impulsive flare phase. As mentioned above, Aurass et al. (2002) and Aurass \& Mann (2004) gave the first radio evidence for the TS that should be formed during the reconnection process. In the EOI the coincidence of the TS feature in the spectrum with a $\gamma$-ray pulse released by relativistic electrons is evident (see Table 2 and the white bar in Fig. 2).
- A main phase decimeter (dm) continuum formed in the lowest corona (200-1200 MHz). The estimated proton release time (Table 1) $11 \mathrm{~min}$ after the impulsive flare phase gives 11:12-11:19 from onset to maximum of the GLE-exciting relativistic protons, which is a surprising coincidence with the duration of fine structure emission (fiber or intermediate drift bursts, e.g., Elgaroy 1973; Benz \& Mann 1998) in this - and only in this - dm continuum during the EOI.

We present evidence for their relation with the acceleration of nonthermal particles in two well separated, distinct regions of 


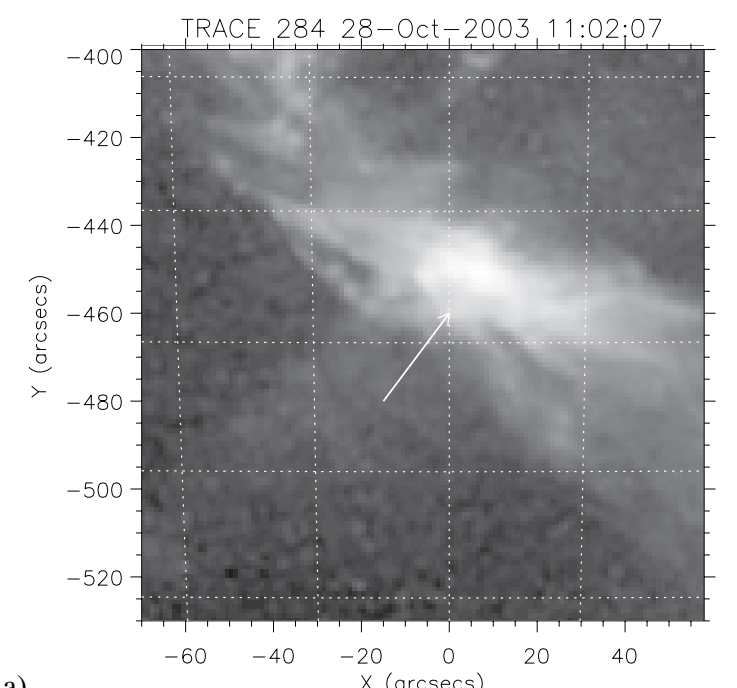

a)

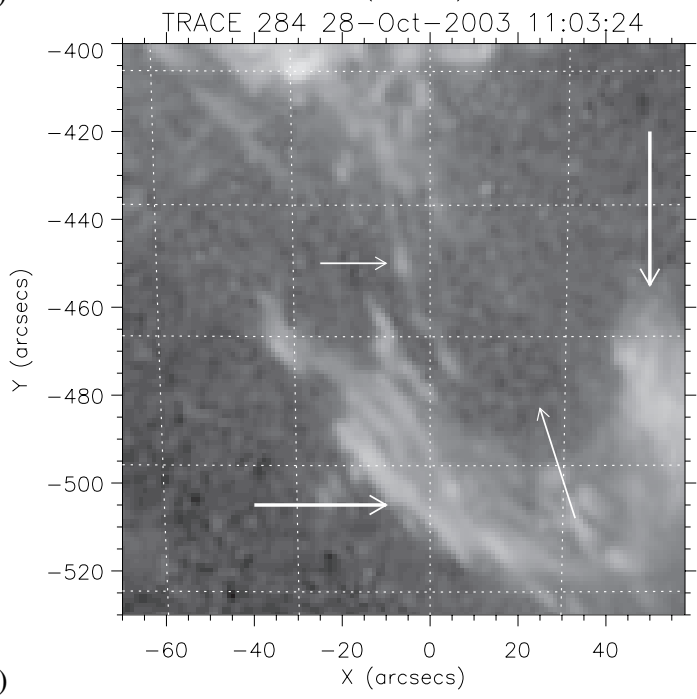

Fig. 3. Erupting features (bright) seen in TRACE $284 \AA$ just before (11:02:07 UT, a)) and during (11:03:24 UT, b)) TS radio emission. Note the fine threads extending from the knot (arrow) in a) and the thin loop (thin arrows) underneath the ejected bright matter (thick arrows) in b). The ejection moves with $\approx 1080 \mathrm{~km} \mathrm{~s}^{-1}$ in the plane of sky.

the corona. Both can be key features to understanding the association between delayed particle injections and related low corona and surface effects, respectively. This association was not well understood in the given case (Klassen et al. 2005) and in many others (Kahler et al. 2006, for Ref.).

\subsection{The outflow termination shock radio signature}

The radio spectrum of the EOI and the corresponding NRH imaging data are already shown by Pick et al. (2005) so that we can concentrate our attention on some important details concerning the onset and the very first minutes of the impulsive phase (the time between 11:01 and 11:04 UT). This time interval is of special interest because Gros et al. (2004) found a well-defined isolated $\gamma$-ray enhancement in the $\geq 7.5-10 \mathrm{MeV}$ range interpreted as bremsstrahlung from relativistic electrons. The time range of this effect is marked in Fig. 2, and we contrast it in this figure with a special feature in the AIP radio spectrogram. The spectrum shows many characteristics of type II burst emission. From the $140-170 \mathrm{MHz}$ part, we recognize that there is dominantly harmonic mode emission between 200 and $500 \mathrm{MHz}$.

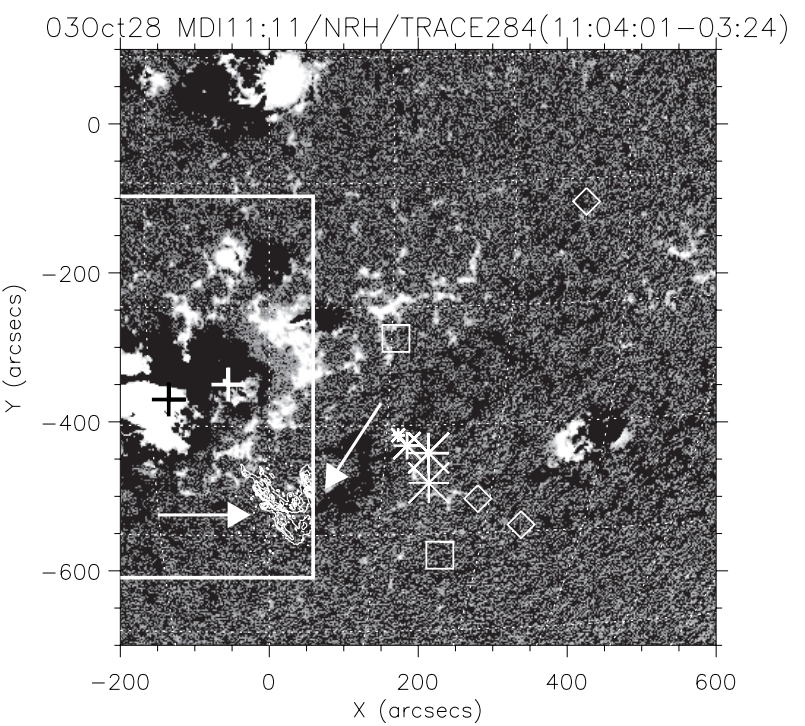

Fig. 4. SOHO-MDI magnetogram and several radio source sites (from $\mathrm{NRH}$ ) related to Figs. 2 and 3. Asterisks (smaller to larger): the TS source at 432, 410.5, and $327 \mathrm{MHz}$; halfpower source diameter $\approx 200$ arcsec. Squares: $327 \mathrm{MHz}$ sources during the neg. drifting branch of the TS. Diamonds: $236.6 \mathrm{MHz}$ sources during TS. Large box: W-part of the TRACE FOV. White isolines (arrows): $284 \AA$ difference image 11:04:01-11:03:24 UT. Crosses in the TRACE FOV: RHESSI sources 11:06 UT, for reference.

This is independently confirmed by the Artemis spectrometer (courtesy: X. Moussas). It is, according to our present experience, the first case of a TS signature with a clear fundamentalharmonic $(\mathrm{F}-\mathrm{H})$ pattern in the spectral data. A detailed description of its properties is given in Appendix A.

We found a looplike feature inclined toward SW in TRACE $284 \AA$ images (1.25-4 Mio. K) simultaneously with the TS signature in the spectral data. Ahead (more SW-ward) of this probably prominence-related hot and dense plasma (Fig. 3a, 11:02:07 UT, arrow), an X-shaped pattern formed by bright elongated threads occurs. Later (Fig. 3b, 11:03:24 UT), the Xshaped pattern has left the TRACE FOV, and we see expanding bright matter (bold arrows) only, with a faint indication of a somewhat streched closed loop behind (thin arrows). A projected rise speed of about $1080 \mathrm{~km} \mathrm{~s}^{-1}$ was estimated near the ejection's leading edge. Given the time sequence of TRACE images, the lift-off of the discussed feature is between 11:02:31 and 11:02:47 UT, which is just the time of the pulse onset of $\geq 7.5-10 \mathrm{MeV} \gamma$-ray emission and the start of the TS signature in radio (Fig. 2, $\approx 11: 02: 40 \mathrm{UT}$, and Table 2).

In Fig. 4 we overplotted the radio source centroids during the high frequency herringbone (HB) emission of the TS source (see Fig. 2) on the MDI magnetogram. From smaller to larger asterisks, the centroid position of the 432, 410.5, and $327 \mathrm{MHz}$ source between 11:02:45 and 11:03:20 UT is plotted. The sources are ordered in frequency, with the highest frequency next to the active region. Squares give the $327 \mathrm{MHz}$ source later (11:03:39), during the negative drift tendency of the second harmonic backbone in Fig. 2. The southern source moves with some scatter further to south and dominates the $327 \mathrm{MHz}$ event flux again between 11:50 and 12:05 UT (Sect. 3.3). Diamonds give the source centroid positions obtained at $236.6 \mathrm{MHz}$ in the interval 11:03:45-50 UT. The loop-like bright feature east of the TS radio source (arrows) is the TRACE $284 \AA$ difference image (11:04:01-11:03:24 UT), plotted here as white isolines. 
From Fig. 4 we get a (projected) distance between the TS radio source and the rising feature seen by TRACE at 11:04:01 UT of $\approx 170 \operatorname{arcsec}\left(124 \mathrm{Mm}\right.$ or $\left.0.18 R_{\odot}\right)$. In projection, this rising structure passes eastward of the TS radio source centroid. It is tempting to assume that the drift rate modulation of the TS burst backbone is somehow related to the passing of this hot and dense magnetoplasma. The onset of a fast rise motion in the flaring active region is important for understanding the growth of a termination shock at the edge of a (counterstreaming !) outflow jet.

Concluding, we remind the reader again that the TS feature in the radio spectrogram was recorded simultaneously with the pulse of $\gamma$-ray emission (Fig. 2, white bar). We argue that in the given event, the TS was an efficient accelerator of flare electrons early in the impulsive flare phase, acting during the filament eruption as observed by TRACE (Fig. 3). Mann et al. (2006) presented, using the EOI parameters, a fully relativistic treatment of particle acceleration at shocks in the TS geometry. They underline the essential role of the standing fast mode wave in flare energy release as suggested by the presented observations.

\subsection{The $d m$ continuum at a new source site, during relativistic proton injection}

As already noticed in Table 2, nonthermal radio emission was observed during the main flare phase, too. In Fig. 5, the radio spectrum of the EOI is shown with gaps between 14-40 and $100-200 \mathrm{MHz}$ for the time interval 11-12 UT. The spectra show the impulsive phase till $\approx 11: 10 \mathrm{UT}$, and several revivals of nonthermal dm-m-Hm emission thereafter. The WIND spectrum characterises the extent of the radio sources up to about $14 R_{\odot}{ }^{5}$. It shows an emission interruption in the range between 10 and 14 MHz from 11:22-11:25 UT. Earlier, we see the low-frequency broad-band continuation of impulsive phase fast drift bursts and flare continuum there (Robinson 1985). This is followed by low frequency type II burst F-H-lanes after 11:10 UT. At 11:25 UT, the WIND spectrum changes its character. A continuum emission occurs, extending from the meter wave range, with a slowly decreasing low-frequency cut-off. This continuum fluctuates with a time scale of minutes, and only between 11:58 and 12:05 UT do new fast drift bursts reach lower frequencies exceeding the continuum low-frequency limit. However, these late fast drift bursts do not cover the full WIND-RAD1 bandwidth, in contrast with the bursts before 11:22 UT.

The radio emission considered is due to the low energy component of nonthermal electrons (say the range between 1 and $10 \mathrm{keV}$ ). What makes the low frequency cut-off distinction between the WIND radio signatures before and after the interruption at about 11:22 UT? We argue that the two different electron injections (see Table 1) before and after 11:25 UT are faced with different escape conditions. They probably come from two different acceleration sites.

The decimeter continuum from 11:12 until $\approx 11: 20$ UT occurs just in the time in which the release of the GLE-inducing relativistic protons starts and reaches its maximum (Klassen et al. 2005). Further, it terminates with the broad band features extending over the whole WIND-RAD1 bandwidth (Fig. 5). The $\mathrm{dm}$ continuum also deserves attention because of two other independent findings:

- During this continuum, and in none of the other broad band enhancements in the EOI, patches of fiber bursts (or

\footnotetext{
${ }^{5}$ Using the density model of Mann et al. (1999).
}
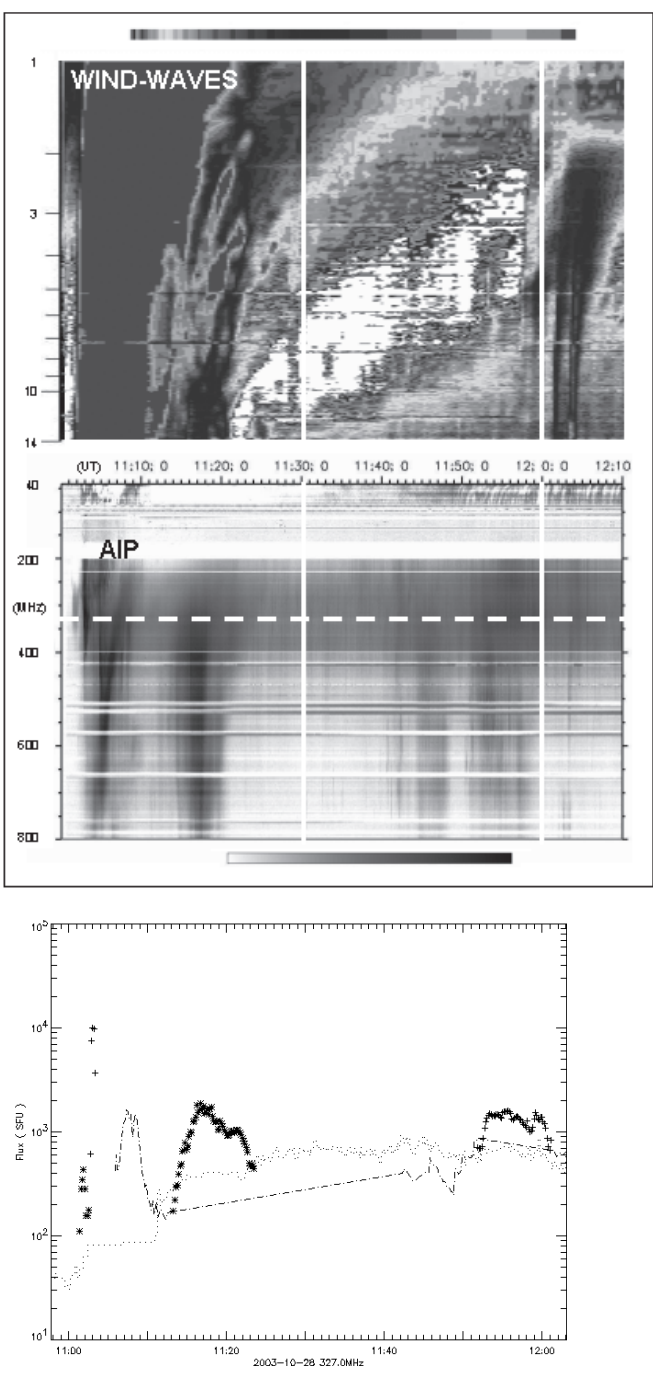

Fig. 5. Top: WIND-WAVES spectrum, range 1-14 MHz (courtesy: M. Kaiser), log. scale, and AIP 40-800 MHz, lin. scale, 11-12:10 UT. Bottom, upper envelope: flux of the strongest NRH source at $327 \mathrm{MHz}$ (cut at the dashed white line in the AIP spectrum). Asterisk: the source with fine structures during onset to maximum of relativistic proton injection. Plus \& dash-dot-line: the TS source; dots: sources above AR 10486.

intermediate drift bursts) occur in the range of 400 to $1200 \mathrm{MHz}$ (Fig. 6).

- The continuum comes mainly from a newly formed source site north of AR 10486 (Figs. 5, bottom, and 7). In the very beginning $(\approx 11: 13 \mathrm{UT})$, some fibers can be localized above AR 10486, too.

Fiber bursts are a radio spectral fine structure occuring in some type IV radio bursts. It is unknown why they sometimes appear and sometimes do not. Benz \& Mann (1998) discuss competing approaches of explaining fiber bursts. Aurass et al. (2005) used the whistler wave model of fiber bursts to determine the coronal magnetic field in postflare loops, thus underlining whistler waves propagating along the coronal magnetic field are a very probable exciter of fiber bursts (see also Vocks \& Mann 2006). Following this model, whistler waves are driven by a loss-cone distribution of relativistic electrons trapped in coronal loops.

In the EOI, patches of fibers occur initially $(\approx 11: 13$ UT) in the range $300-800 \mathrm{MHz}$. In the next two minutes, untill 11:15 UT, the range of fiber occurrence is shifted toward higher 

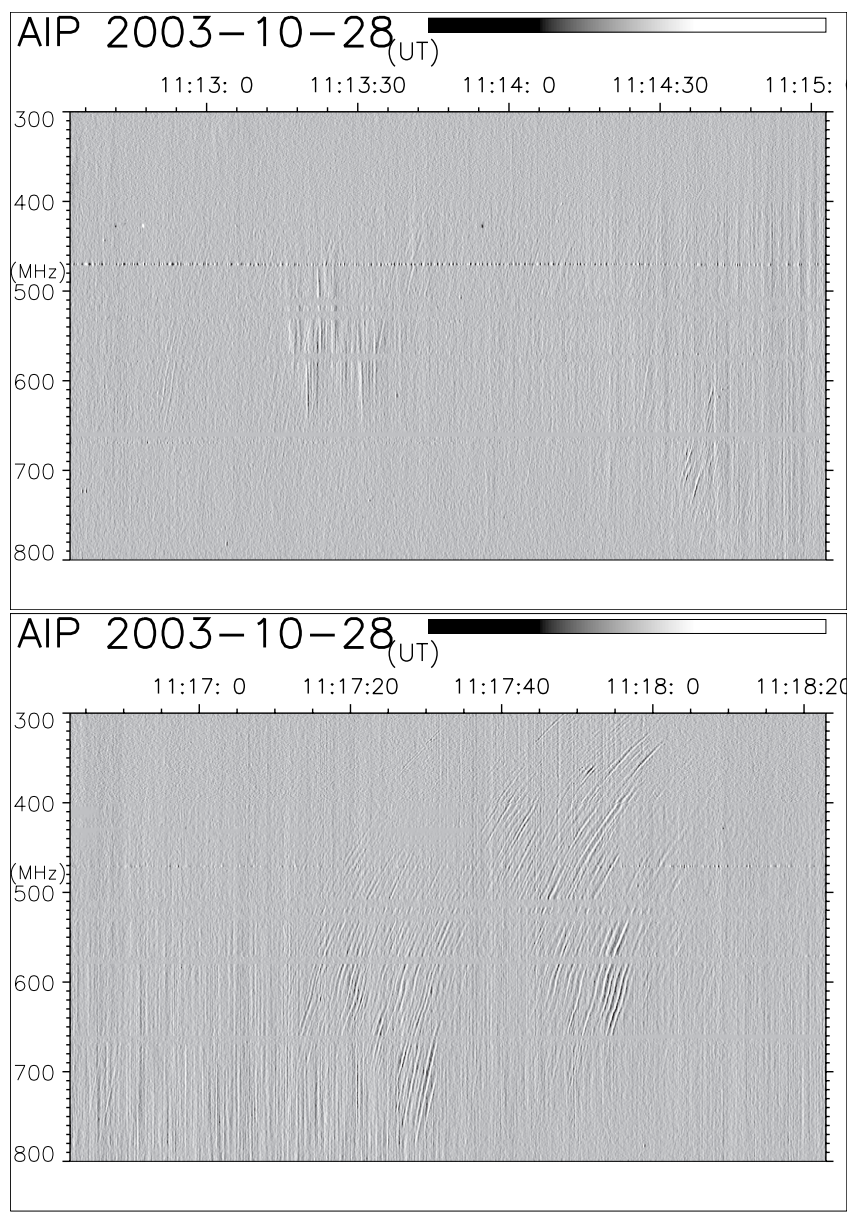

Fig. 6. Some fiber bursts during the continuum enhancement 11:12 until $\approx 11: 20$ UT (time derived dynamic spectra). The nondrifting pulsating features are above the frequency range of NRH and are not considered here.

frequencies (800-1200 MHz, AOO spectral data). Later, at $\approx 11: 18 \mathrm{UT}$, the fibers return back into the range $300-800 \mathrm{MHz}$ and are more intense than in the beginning (see Fig. 6). As mentioned above, the continuum emission with fibers comes from a well-defined isolated source that sometimes dominates the flux of the radio event. The $327 \mathrm{MHz}$ flux curve is shown in Fig. 5 at the bottom. The source N of AR 10486 is brightest between 11:12 and 11:22 UT (asterisks). It is visible neither earlier nor later at other frequencies; only the NRH $327 \mathrm{MHz}$ record reveals a short flash-up earlier (at 11:02-03 UT), confirmed by the narrowband feature in the AIP spectrogram (Fig. 5, top).

In Fig. 7, we have overplotted the sites of the radio sources dominating the event in different time intervals between 11 and $12 \mathrm{UT}$ at 327 and $410 \mathrm{MHz}$, respectively, on KSO $\mathrm{H} \alpha$ blue wing and SOHO-EIT $195 \AA$ images. The fiber burst-rich dm continuum appears well north of AR 10486. In the $\mathrm{H} \alpha$ image, we have further inserted descriptors Fx for several filaments of interest in the EOI. A detailed discussion of Fig. 7 is given in Appendix B.

SW of AR 10486 at 410 and $327 \mathrm{MHz}$, Fig. 7 reveals a source site that we have discussed already in Sect. 3.2. It was misleadingly called a type III burst source by Pick et al. (2005). It shows up during the TS in the spectrum and, after some spatial scatter, between 11:05 and 11:10 UT when the spectral signature is less specific. Note that this source reappears once again between 11:55 and 12:05 UT with an unspecific broad-band radio signature, confirming the long lifetime of a m-dm radio source
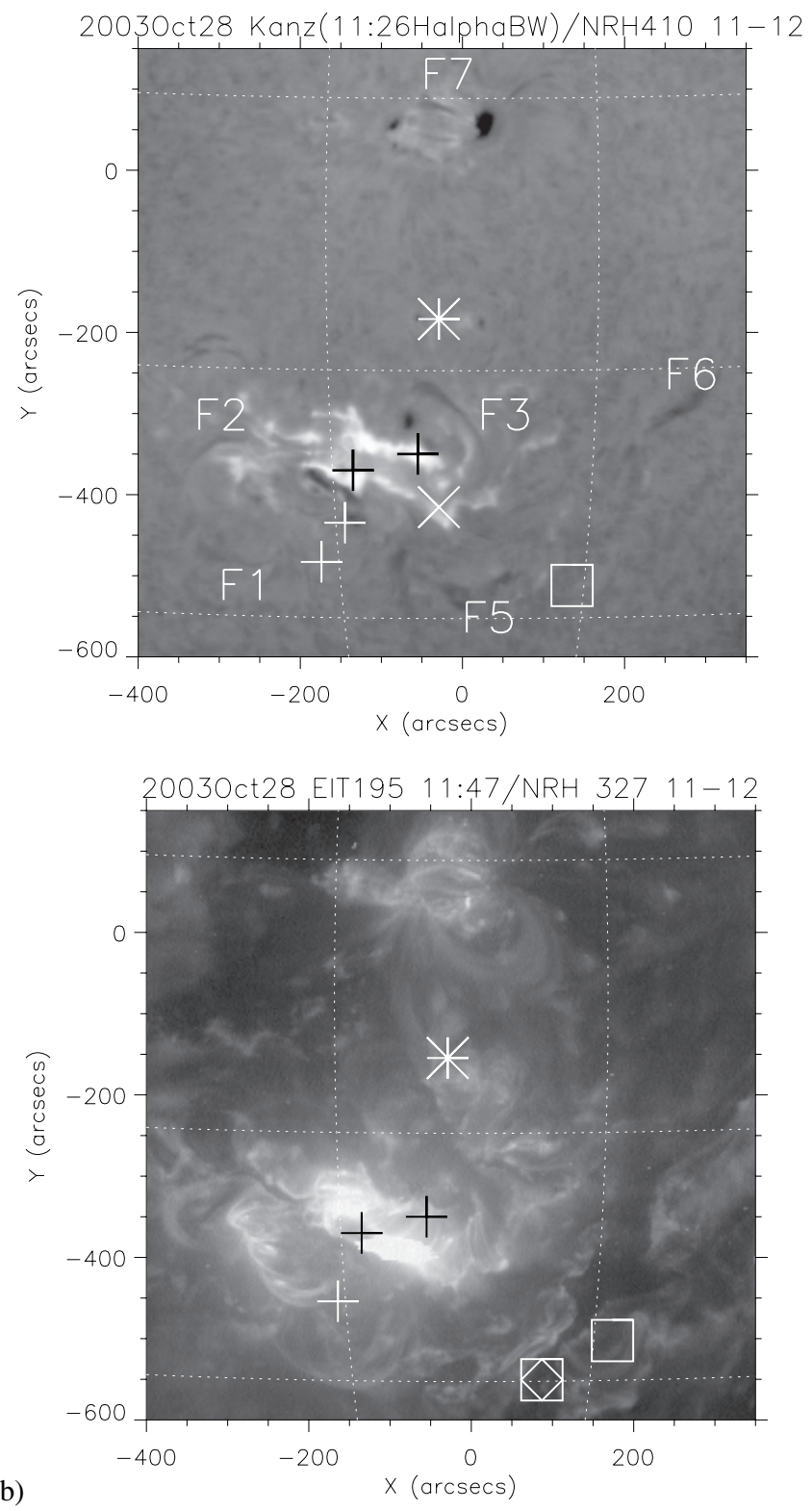

Fig. 7. NRH source sites, overview 11-12:10 UT, $410 \mathrm{MHz}$ (a, overplotted on KSO H $\alpha$ blue wing 11:26 UT) and $327 \mathrm{MHz}$ (b, on EIT$11: 47$ UT). The asterisk $\left(\approx 0.2 R_{\odot} \mathrm{NW}\right.$ of AR 10486) marks the dm continuum source site of special interest here. Squares: TS and continuum sources; white + and X: sources above AR 10486. F1-F3 and F5-F7 filaments. The dark bold + are the 11:06 UT RHESSI HXR sources, for reference.

site at about this place. This is in accordance with the findings of Aurass et al. (2002), who initially recognized the TS source due to its isolated, less involved occurence in some dynamic spectra $\approx 1 \mathrm{~h}$ after the impulsive phase.

We have mentioned above that the whistlers that excite the fiber bursts are formed due to a loss-cone distribution of energetic particles in a magnetic trap. To identify the source of the energetic particles that drive the whistler waves, we check the RHESSI images in the fiber burst time interval. In Fig. 8, we show the HXR sources in the ranges 12-25 keV (black) and $\geq 100-300 \mathrm{keV}$ (white) overplotted on a TRACE image (195 A, 11:12 UT). A thermal source is situated near the apex of an underlying magnetic structure, while the nonthermal sources occur in the footpoints. The HXR sources are displaced from the main 


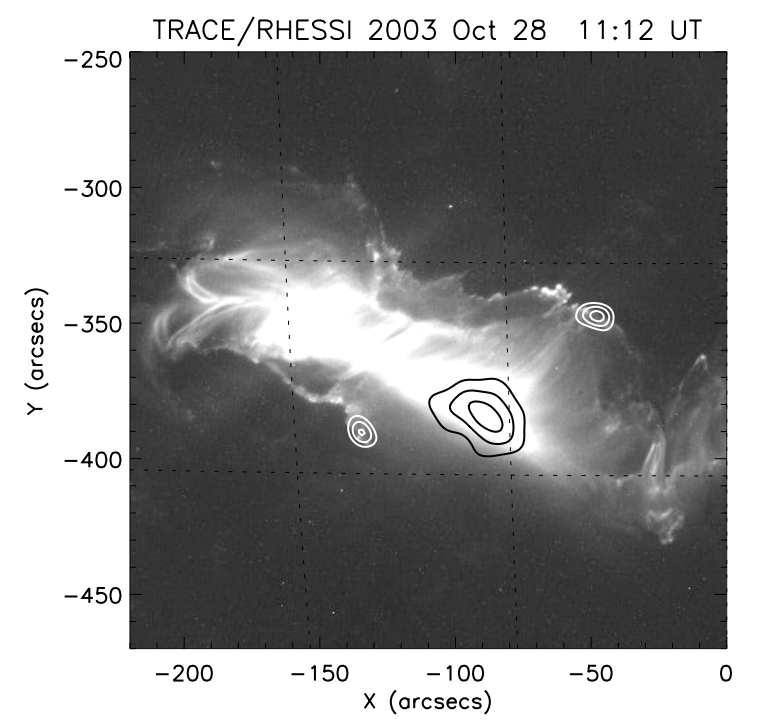

Fig. 8. TRACE $195 \AA$ image taken 11:12:05 UT with superposed (time-integrated 11:12-11:13 UT) RHESSI X-ray source sites. Black: $12-25 \mathrm{keV}$; white: $\geq 100-300 \mathrm{keV}$. The X-ray sources are situated in low-lying postflare loops.

fiber burst-related radio source site, which was found out of the range plotted in Fig. 8 (see Fig. 7). So, we have to check the radio data once again.

Between 11:12 and 11:20 UT there are two intervals in the AIP spectral range with well expressed patches of fiber bursts (Fig. 6). Using the NRH imaging data, and a method described by Aurass et al. (2005), we analyse the relation between the radio source sites of fiber bursts and coronal magnetic field structures. The method yields a bunch of fiber burst-related coronal field lines, which is a subset of the lines obtained by potential field extrapolation of the SOHO MDI 11:11 UT magnetogram. From the ensemble of extrapolated field lines, those lines that pass through a certain surrounding of the source centroid of a fiber burst source ${ }^{6}$ at least at two neighboring NRH frequencies are selected. These field lines enclose the propagation path of the whistler wave packets that excite the fiber burst radio emission. The highest imaging frequency of the NRH is $432 \mathrm{MHz}$. The spectral information at higher frequencies cannot be used for this analysis.

We obtain an interesting result: in the phase between 11:12 and 11:15 UT, the fiber burst patch in the spectrum is composed of fiber bursts from two different sites above the considered group of active regions. One source site is immediately above the flaring region NOAA 10486 (Fig. 9a). The radio source is in the southward branch of a field connection crossing the main flare ribbons and almost coinciding with one of the nonthermal HXR sources, and in a strong flux concentration connected toward $\mathrm{S}$. The other fiber burst source leads to a magnetic junction between the N-part of AR 10486, further towards ARs 10491 and 10488 (Fig. 9b). Near the southern footpoint of this magnetic junction the northern nonthermal X-ray source in AR 10486 (Fig. 8) appears.

In the later phase (11:17-11:19UT, Fig. 6b), only one source site of fiber bursts can be identified which is almost identical, with a slight $\mathrm{W}$ shift to the N-ward connection from AR 10486 to AR 10488 (Fig. 9b).

\footnotetext{
${ }^{6}$ See the black squares in Figs. 9 and 10.
}

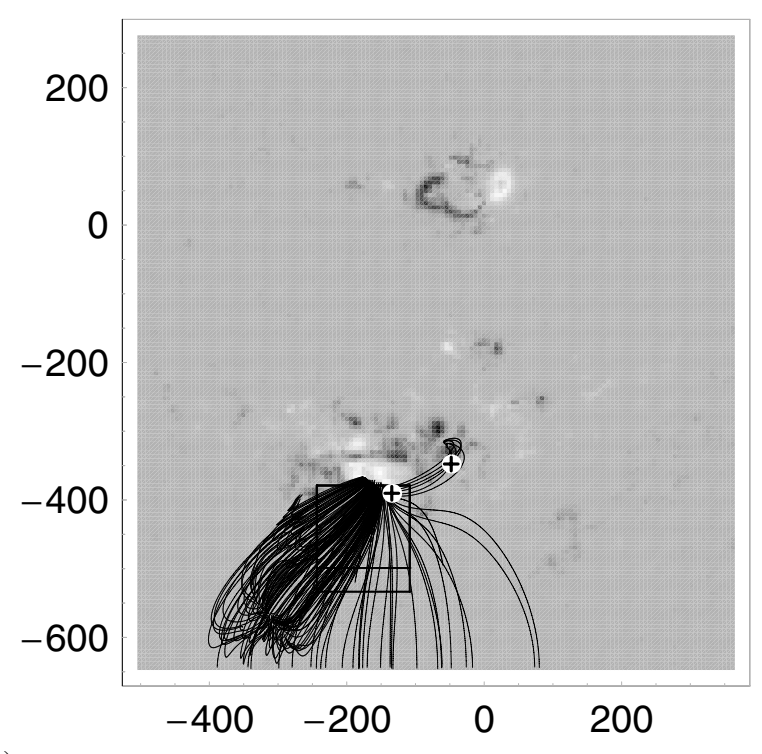

a)

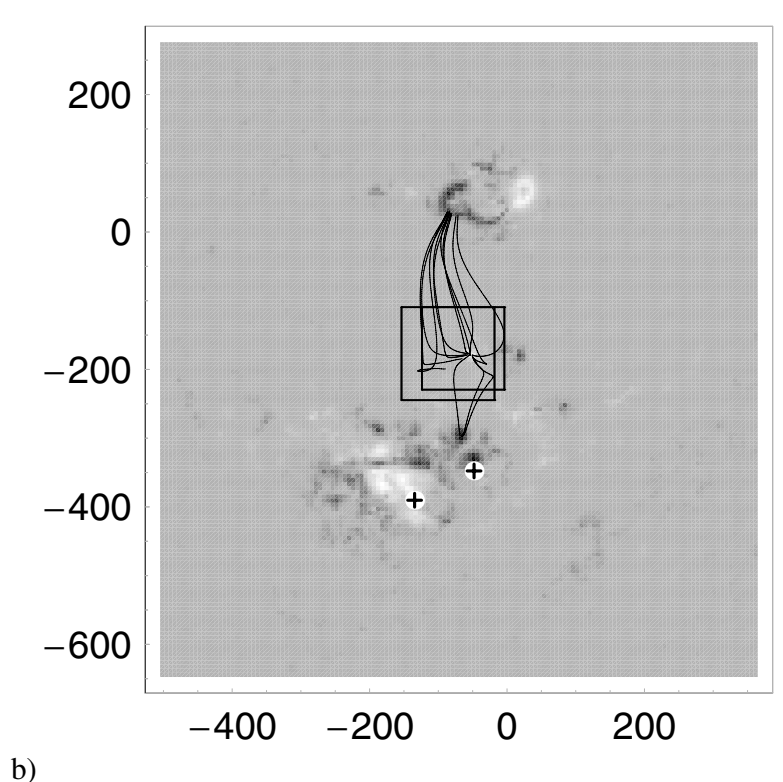

Fig. 9. Field structures (Cartesian potential field extrapolation of SOHO MDI 11:11 UT) selected by the fiber burst sources (black boxes) applying a five-fold Newkirk coronal density model. a) the S-ward situated source site occuring during 11:12-11:15 UT, only. b) the N-ward situated source site observed at 11:12-11:15 and 11:17-11:19 UT. White dots with inserted + are the HXR source centroids from Fig. 8 .

If we apply the spherical potential field extrapolation ${ }^{7}$ we should obtain a more reliable information about large-scale magnetic interconnections. The result shown in Fig. 9a changes into the pattern given in Fig. 10a. Namely, one newly detected magnetic connection joins - in accordance with the fiber radio source - the southern part of AR 10486 with AR 10493. As evidenced by Fig. 10b, this is the only region of open field lines routed in the discussed activity complex.

Accepting the given static representation of the coronal magnetic field we find that the fiber burst sources occur in several closed magnetic field systems that must not be immediately magnetically linked with the HXR- and $\gamma$-ray source sites. This means if the fiber-exciting particles are injected by a low-coronal

${ }^{7}$ Using Schrijver \& DeRosa (2003) in the field line analysis routine. 

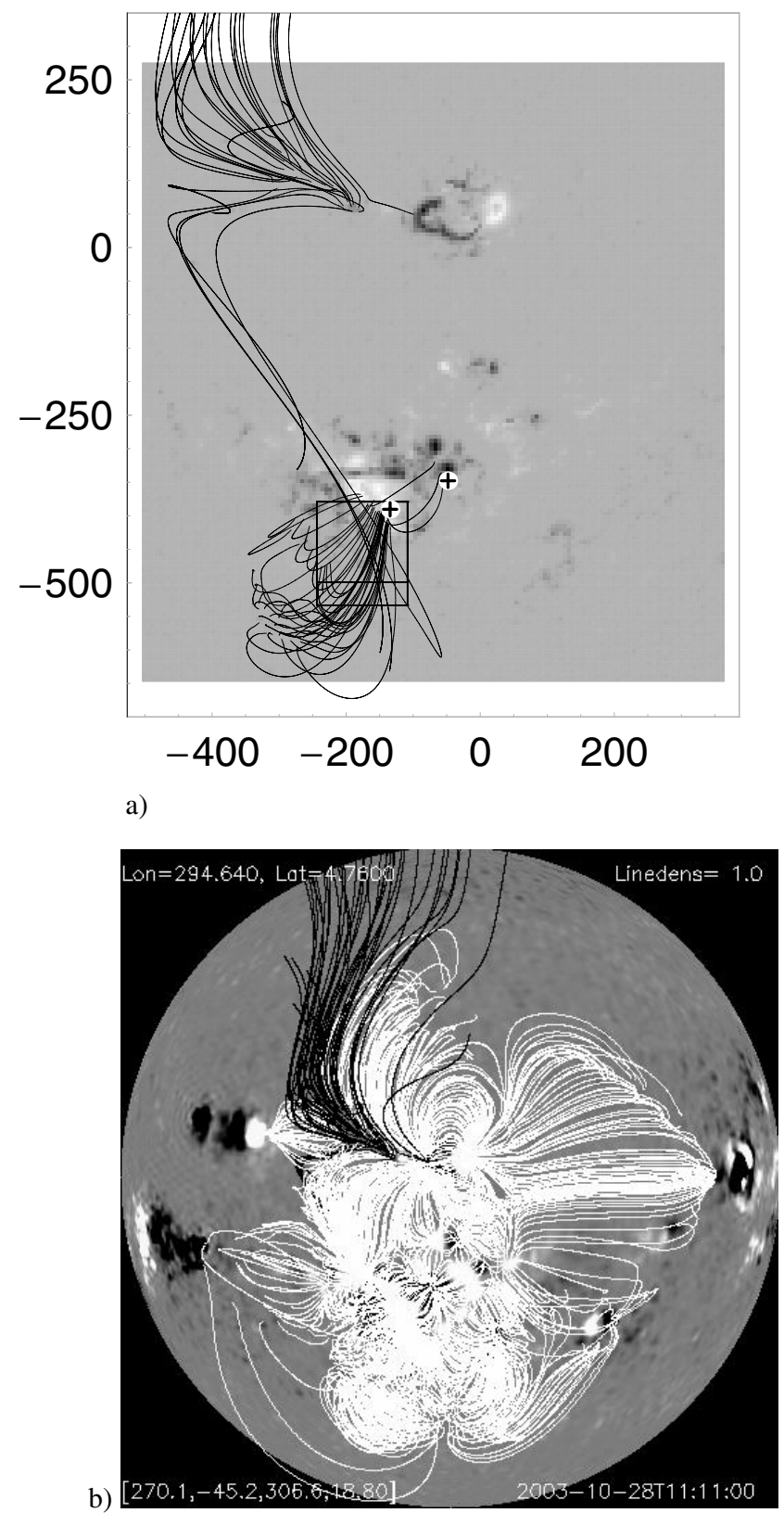

Fig. 10. Field lines of the spherical potential field extrapolation according to Schrijver \& DeRosa (2003), time 12:04 UT $\pm 6 \mathrm{~h}$. a) The selected lines for the fiber source near AR 10486 (11:12-11:15 UT), density model as in Fig. 9. Here, we find a magnetic connection to the footpoint range of a bunch of open field lines in AR 10493. The open lines are added to those selected by the fiber bursts. White dots with +: HXR source centroids from Fig. 8. b) The full set of field lines of the extrapolation used left, for comparison. The closed field lines are drawn white, open lines dark.

accelerator related with the source sites of high energy radiation, these particles can access the remote loops only due to diffusion across field lines in neighbouring footpoints. It turns out that the detected connections in Figs. 9b and 10a form a ring-like configuration which also includes one escape point of trapped particles if they reach the open field line range at AR 10493. In this context we also remind the reader to Fig. 1c and the ring-like separatrix pattern detected in the height range of $70-150 \mathrm{Mm}$ (Sect. 3.1).

\subsection{Giant postflare arches in the corona?}

The qualitative change of the WIND spectrum (from before to after 11:22-11:25 UT) occurs simultaneously with the decay of the proton injection-associated dm continuum with fiber bursts that was discussed in Sect. 3.3. The spectral data shown in Fig. 5 reveal the formation of a new type of continuum emission, which slowly broadens in bandwidth toward lower frequencies. We estimated the radial speed revealed by this frequency $\operatorname{drift}^{8}$ as $30 \mathrm{~km} \mathrm{~s}^{-1}$, for the interval 11:25-12 UT. This fits with observations of the rise speed of coronal postflare giant arches (Švestka 1984). Minute scale fluctuations over a broad frequency range that is well visible at the AIP spectra (between 250 and $40 \mathrm{MHz}$ ) independently confirm the presence of large coronal loops. The NRH 236.6 MHz images reveal a source with min fluctuations above AR 10486 (checked between 13:00-13:30 UT). We argue that the min scale fluctuations are excited by standing magnetoacoustic flux-rope oscillations as reported by Aurass \& Mann (1987) for the huge August 4, 1972 flare.

From $\mathrm{SOHO}$-LASCO-C2, white light corona images are available at 11:30 and 11:54 UT. We processed these images to enhance brightness gradients. The results are shown in Fig. 11. A cusp-shaped substructure, hidden in the background of the evolving fast halo CME (Table 2), becomes visible moving outward with a plane-of-sky speed of $1083 \mathrm{~km} \mathrm{~s}^{-1}$ during the decimeter continuum reactivation and the low frequency bandwidth rise observed by WIND. The streamer cusp top is marked by a cross, and the $327 \mathrm{MHz}$ TS source site as a square on the disc, for reference. The cusp speed coincides with the velocity of the ejection seen in TRACE $284 \AA$ early in the impulsive phase $\left(1080 \mathrm{~km} \mathrm{~s}^{-1}\right)$. We argue that this cusp marks the lower end of the flare current sheet. The flaring magnetic arcade structure is evolving (following the two-ribbon flare scenario, e.g., Priest \& Forbes 2000) and rises beneath the faster escaping halo CME.

During the whole event, a part of the observed coronal structures moves at roughly $1100 \mathrm{~km} \mathrm{~s}^{-1}$. This is contrasted by features moving about three times faster ${ }^{9}$. Fast moving features are indicated by the drifting branches of the TS lane (Fig. 2), the regular low frequency type II bursts (11:10-11:24 UT in WIND-RAD1, Fig. 5), the outer rim of CME-related radio emission (Pick et al. 2005), and the halo CME in LASCO-C2 (after 11:30 UT). They point to speeds between 2500 and $3300 \mathrm{~km} \mathrm{~s}^{-1}$. In discussing the flare-CME relation, it is sometimes not evident how the large current sheet forming behind a CME and the (initially smaller scale) postflare current sheet belong together. Here the LDE flare current sheet seems to be a persistent part of the relaxing post-CME corona.

\section{Discussion}

We analysed several observations during the huge X-class flare on October 28, 2003, to study the flare-CME-particle acceleration puzzle. Our intention was to assemble low corona observations suitable to be compared with the particle injection timing derived by Klassen et al. (2005). We discuss these facts with the aim of understanding more about the sources of accelerated particles.

\footnotetext{
${ }^{8}$ Using the density model of Mann et al. (1999).

9 We estimate a speed measurement uncertainty of about $\pm 100 \mathrm{~km} \mathrm{~s}^{-1}$ in the plane of the sky, and due to the frequency drift-speed transformation.
} 

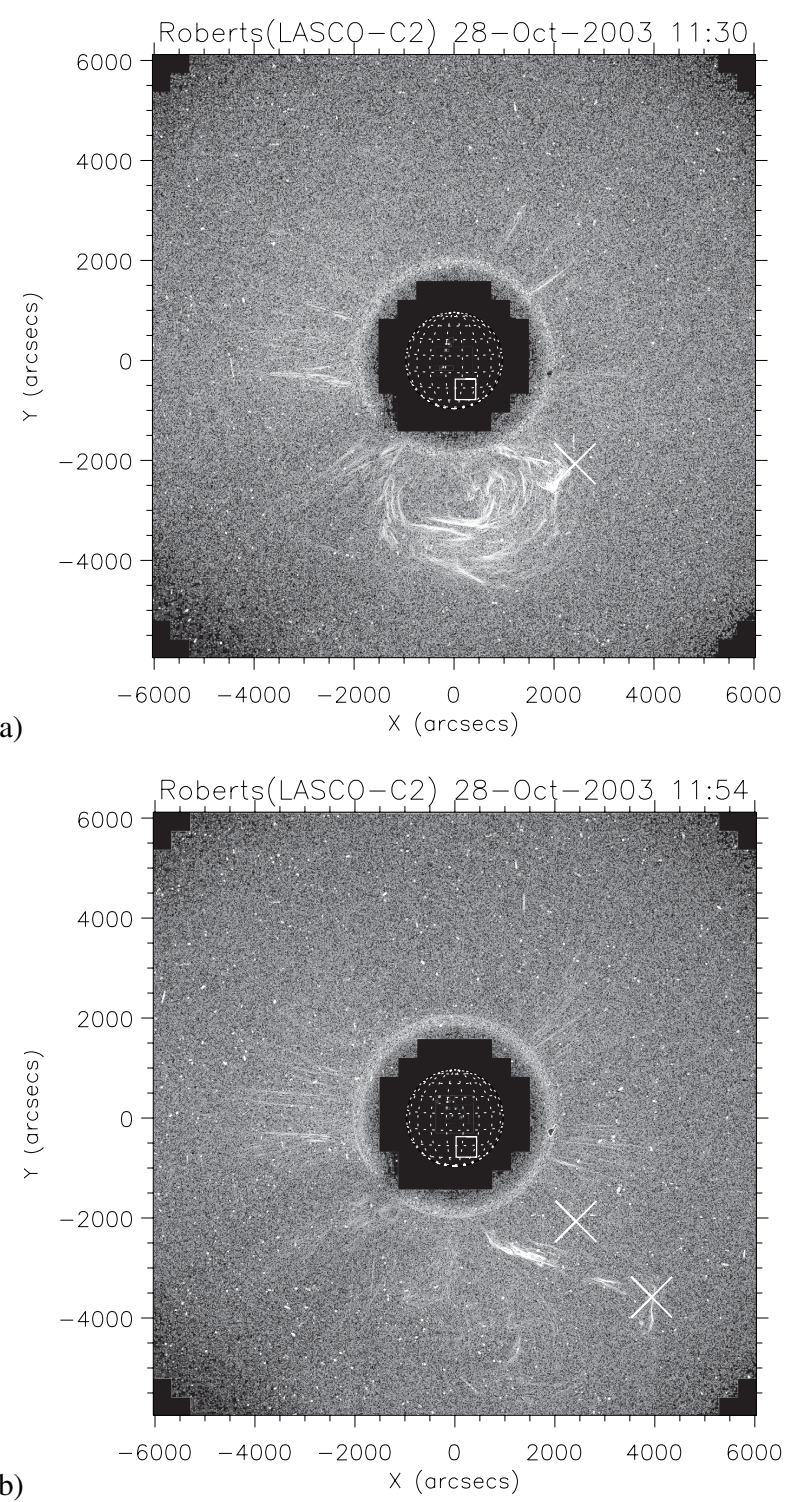

Fig. 11. $S O H O$-LASCO-C 2 halo $\mathrm{CME}$ images, contrast-enhanced, a) 11:30 and b) 11:54 UT. The box on the disc is the long-living $327 \mathrm{MHz}$ source, see Figs. 5 and 7. Crosses in both panels mark the top of a rising cusp, in b) the cross of a) is also redrawn.

There are (at least) three situations possible: particles are accelerated

- during a flare, as in the confined and arcade flare scenario, with magnetic reconnection as an important ingredient;

- as a byproduct of the opening and reclosure of the coronal magnetoplasma-configuration during the release of a CME; and

- at a coronal and/or an interplanetary shock wave.

In all cases the accelerated particles must stream back toward the photosphere to become indirectly visible by X-ray or $\mathrm{H} \alpha$ emission. The accelerated particles may possibly become trapped in closed magnetic configurations thus leading to nonthermal radio continuum emission, sometimes showing radio spectral fine structures. An azimuthal propagation of these particles perpendicular to the magnetic field lines is possible only in the regions of higher collision rate near the footpoints of closed magnetic configurations. Hudson \& Ryan (1995) indicate reports of "large area $\gamma$-ray flares", which demonstrate proton acceleration and transport involving large parts of the corona including multiple magnetic structures.

In the EOI, the first impulsive $\gamma$-ray emission is due to relativistic electrons accelerated at the TS. As already suggested by Mann et al. (2006), a corresponding acceleration mechanism can act with sufficient efficiency under coronal conditions. The TS radio source occurs, in projection, $\mathrm{SW}$ of the flaring active region. The TS spectrum shows drift rate modifications during approach, bypassing, and drifting apart of hot and dense matter from the impulsive phase filament eruption. The frequency drift rate modifications in the TS feature during simultaneous fast liftoff motions yields an additional argument for the assumption that this spectral signature is related to shock formation in the descending reconnection outflow jet.

The TS source site fits with LASCO-C2 coronograph images of the fast halo CME. The former TRACE-284 $\AA$ ejection is still persisting and now reappears in the white light coronograph data. It seems to belong to the southern boundary of a large helmet streamer above giant postflare arches. In accordance with Cliver et al. (1986) we argue that the helmet streamer current sheet on top of the observed cusp feature is the source of the "gradual" high energy electron stream reported by Klassen et al. (2005).

The relativistic protons, detected as GLE (Miroshnishenko et al. 2005) $)^{10}$, are accelerated during a decimeter continuum source. The corresponding radio source centroid is situated well N of NOAA 10486, above the smaller region 10491, and near a magnetic separatrix in the extrapolated potential magnetic field. Trottet et al. (1994) and Aurass et al. (1998) have already noticed the occurrence of an additional remote radio source site during $\gamma$-ray events. As shown above, during the EOI this radio source site can be reached by particles of the ensemble feeding the sources of high energy emission. So in the EOI, the whistler waves exciting the fiber burst radio emission can be lowfrequency waves in resonance with energetic protons. Then, the spatial extent of proton-related magnetic substructures fits with the "large area $\gamma$-ray flare" phenomenon mentioned by Hudson \& Ryan (1995). We have shown that magnetic paths leading to the only open field gap of the (static) magnetic field configuration existed, accepting the spherical potential field extrapolation. In such a case the exclusive presence of the fiber burst fine structure in the analysed dm continuum, at a specific source site during the complex evolution of the EOI, independently proves the presence of energetic protons in the lowest coronal structures on the disk, simultaneously with their injection onset as was back-extrapolated from neutron monitor data. This makes a low-corona acceleration site very probable, although it is difficult to explain it with the acceleration of particles at a remote travelling shock wave.

\section{Conclusions}

The injection times of various components of energetic particles were derived by Klassen et al. (2005) for the October 28, 2003, $\mathrm{X}$-class/ $\gamma$-ray flare in NOAA AR 10486. This flare occurred in connection with a fast halo CME and a GLE. We used radio (AIP, WIND, NRH), H $\alpha$ (KSO), RHESSI, SOHO (EIT, LASCO, MDI), and TRACE data to study the associated chromospheric and low coronal phenomena. Mainly guided by spectral and imaging information about the nonthermal radio emission, and the potential field extrapolation of the longitudinal component of the

\footnotetext{
${ }^{10}$ See this paper also for a discussion of the connectivity problem.
} 
photospheric magnetic field, we tried to identify the solar sources of the different particle ensembles. In this event, three source sites of accelerated particles can be discriminated:

1. a stable source, in projection $0.3 R_{\odot}$ away of AR 10486, which we identify as the site of the reconnection outflow termination by the TS signature in the dynamic radio spectrum in the impulsive flare phase.

2. The extended current sheet above a giant coronal postflare loop system, in the given case a part of the erupting flux rope flare/CME scenario and of the post-CME coronal relaxation, in the main flare phase.

3. A source situated on a magnetic separatrix surface between several magnetic arcades and neighbouring active regions, which are more or less included in the onset of the main flare phase, and act during onset and growth of high energy proton injection in space. It is not clear if this source is directly related to the acceleration of protons or if it only points to an energetic particle path between this site and the main $\mathrm{HXR} / \gamma$-ray sources leading to the penetration of energetic protons into a multistructure magnetic loop system, as was already reported for "large area $\gamma$-ray flares" (Hudson \& Ryan 1995).

Our result, for the analysed event, is in favour of energetic particle acceleration in the low corona $\left(<0.5 R_{\odot}\right.$ above the photosphere) and in contrast with acceleration of the relativistic particles at remotely propagating shock waves.

Acknowledgements. The authors kindly acknowledge the use of the different instruments and data pools that are explicitely mentioned in Sect. 2. Further, they are grateful to Dr. A. Hofmann for running the field extrapolation software (Cartesian coordinates) and for advice in data selection. G.R. is supported by Grant No. AU 106/13-1 of the Deutsche Forschungsgemeinschaft. The work of A.W. was supported by DLR under grant No. 50QL0001. The AIP acknowledges the European Office for Aerospace Research and Development for its support in maintaining the solar radio spectral observations. The authors thank Dr. S. Kahler, and the referee, Dr. S. Krucker, for their careful reading and helpful comments on the manuscript.

\section{Appendix A: Detailed description of Fig. 2}

The TS feature shown in Fig. 2 has several remarkable properties.

There is a band split type II backbone starting at 11:02:37 with a width of $\approx 30 \mathrm{MHz}$ at $250 \mathrm{MHz}$, which is $12 \%$, quite a typical type II burst value (e.g., Vršnak et al. 2001). The band split rises within some seconds to values $\geq 50 \mathrm{MHz}$ (20\%). From the lane, a system of two different sets of herringbone fine structure (HB, e.g., Mann \& Klassen 2005) escapes with drift rates of $\approx 19$ and $11 \mathrm{MHz} \mathrm{s}^{-1}$ yielding speeds between 30000 and $15000 \mathrm{~km} \mathrm{~s}^{-1}$ with the Newkirk (1961) coronal density model. We mainly see the branches toward high frequencies in the figure (note the AIP spectral gap that is between 200 and $170 \mathrm{MHz}$ ).

For about $15 \mathrm{~s}$ the backbone (or better, the starting frequency of HBs) shows a positive frequency drift followed by a further $15 \mathrm{~s}$ of zero drift, changing at 11:03:10 UT toward a negative frequency drift. This corresponds, assuming a small source of plasma emission, to an initial motion toward somewhat higher densities, a stay of about $15 \mathrm{~s}$ there, and then a motion toward lower densities. In the third phase, the wellordered $\mathrm{HB}$ emission becomes more and more chaotic. On average, over all three time intervals, the resulting drift rate of the feature is zero. Transforming the drift rates into speeds using the Newkirk (1961) coronal density model, we obtain an initial speed of $2520 \mathrm{~km} \mathrm{~s}^{-1}$ toward higher densities; and after the $15 \mathrm{~s}$ break (during which the compact $432 \mathrm{MHz}$ source starts to separate into a double source), a $2600 \mathrm{~km} \mathrm{~s}^{-1}$ motion toward lower densities.

The HB pattern is imaged by the NRH at the 3 highest frequencies $(432,410.5,327 \mathrm{MHz})$ showing a relatively compact source, about $0.3 R_{\odot} \mathrm{SW}$ of the flare site (see Fig. 5 in Pick et al. 2005, who improperly call the HBs type III bursts). The source centroids are ordered, with the highest frequency next to the flare site, but only slightly shifted against the next lower frequency.

At the high frequency edge of the spectrum, between 11:03:00 and 11:03:35 UT, a set of narrowband positively drifting elements intermittently occurs, shifted to falling frequency parallel to the dashed white line. This is unusual for type II radio bursts and occurs only during the sudden kink of the gross spectral feature to lower frequencies.

\section{Appendix B: Detailed description of Fig. 7}

The filaments F2 and F3 remain stable during the whole event. In between there is an active region filament along the neutral line, which erupts during the impulsive phase near the northward kink to F3. The $\mathrm{H} \alpha$ image shows faint kinked flare ribbons there, and at the same position there is a source in the $410 \mathrm{MHz}$ image (white X). The long prominence F6 changes only its length (visibility ?) during the flare; at its southern end F5 is near to where the main active region filament erupts ${ }^{11}$. A comparison with Fig. 1d reveals that the impulsive phase filament eruption occurs just in the direction where the overlying magnetic flux density of the coronal field is very low. The filaments F7 and F1 get activated at the time of the dm continuum revivals after 11:40 UT, and eventually F1 erupts at 12:20 UT. The filament F1 is situated in a complex arcade at the SE boundary of AR 10486, together with a $410 \mathrm{MHz}$ and $327 \mathrm{MHz}$ source (white + in Fig. 7), acting early in the impulsive phase and after 11:50 UT. Faint flare ribbons are seen in the $\mathrm{H} \alpha$ image there. The main $\mathrm{H} \alpha$ flare ribbons are situated NW of this arcade. Roughly speaking, they connect the locations of the stable filaments F2 and F3. Both stable filaments fit with potential field arcades (Fig. 1b).

\section{References}

Aurass, H., \& Mann, G. 1987, Sol. Phys., 112, 359

Aurass, H., \& Mann, G. 2004, ApJ, 615, 2004, 526

Aurass, H., Hofmann, A., \& Urbarz, H. W. 1998, A\&A, 334, 289

Aurass, H., Vršnak, B., \& Mann, G. 2002, A\&A, 384, 273

Aurass, H., Rausche, G., Mann, G., \& Hofmann, A. 2005, A\&A, 435, 1137

Benz, A. O., \& Mann, G. 1998, A\&A, 333, 1034

Bieber, J. W., Clem, J., Evenson, P., et al. 2005, GRL, 32, 302

Bougeret, J.-L., Kaiser, M. L., Kellogg, P. J., et al. 1995, Space Sci. Rev., 71, 231 Brueckner, G. E., Howard, R. A., Koomen, M. J., et al. 1995, Sol. Phys., 162, 357

Classen, H. T., \& Aurass, H. 2002, A\&A 384, 1098

Classen, H. T., Mann, G., Klassen, A., \& Aurass, H. 2003, A\&A, 409, 309

Cliver, E. W., Dennis, B. R., Kiplinger, A. L., et al. 1986, ApJ, 305, 920

Delaboudiniere, J.-P., Artzner, G. E., Brunaud, J., et al. 1995, Sol. Phys., 162, 291

Démoulin, P., Bagalá, L. G., Mandrini, C. H., Hénoux, J. C., \& Rovira, M. G. 1997, A\&A, 325, 305

Elgaroy, O. 1973, in ed. J. Delannoy, \& F. Poumeyrol, Proc. 3rd CESRA Meeting, Bordeaux-Floirac, 174

Gopalswamy, N., Barbieri, L., Lu, G., Plunkett, S. P., \& Skoug, R. M. 2005, GRL, 32, 304

Gros, M., Tatischeff, V., Kiener, J., et al. 2004, in Proceedings of the 5th INTEGRAL Workshop, ed. V. Schönfelder, ESA SP-552, et al., 669

11 Not observed in $\mathrm{H} \alpha$ due to bad conditions at KSO, but in TRACE 284 Å, Fig. 3 . 
Handy, B. N., Acton, L. W., Kankelborg, C. C., et al. 1999, Sol. Phys., 187, 229

Haggerty, D. K., \& Roelof, E. C. 2002, ApJ, 579, 841

Hiei, E., Hundhausen, A. J., \& Sime, D. G. 1993, GRL, 20, 2785

Hudson, H., \& Ryan, J. 1995, ARA\&A, 33, 239

Hundhausen, A. J. 1998, in The Many Faces of the Sun, ed. K. Strong, et al. (Berlin: Springer), 143

Jiřička, K., Karlický, M., Kepka, O., \& Tlamicha, A. 1993, SPh, 147, 203

Kahler, S. W., Aurass, H., Mann, G., \& Klassen, A. 2006, ApJ, submitted

Kiener, J., Gros, M., Tatischeff, V., \& Weidenspointer, G. 2006, A\&A, 445, 725

Kerdraon, A., \& Delouis, J. 1997, in Coronal Physics from Radio and Space

Observations, ed. G. Trottet (Heidelberg: Springer), 192

Klassen, A., Krucker, S., Kunow, K., et al. 2005, JGR, 110, A09S04

Klein, K.-L., Krucker, S., Trottet, G., \& Hoang, S. 2005, A\&A, 1047

Krucker, S., Larson, D. E., Lin, R. P., \& Thompson, B. J. 1999, ApJ, 519, 864

Lin, R. P., Dennis, B. R., Hurford, G. J., et al. 2002, Sol. Phys., 210, 3

Mann, G., Aurass, H., \& Warmuth, A. 2006, A\&A, submitted

Mann, G., Aurass, H., Voigt, W., \& Paschke, J. 1992, ESA SP-348, 129

Mann, G., Classen, H. T., \& Aurass, H. 1995, A\&A, 295, 775

Mann, G., Jansen, F., MacDowall, R. J., Kaiser, M. L., \& Stone, R. G. 1999, A\&A, 348, 614
Melrose, D. B., in Solar Radiophysics, ed. D. J. McLean, \& N. R. Labrum (Cambridge: Cambridge University Press), p. 177

Miroshnichenko, L. I., Klein, K.-L., Trottet, G., et al. 2005, JGR, 110, A09S08

Nelson, G. S., \& Melrose, D., in Sol. Radiophysics, ed. D. J. McLean, \& N. R. Labrum (Cambridge: Cambridge University Press), p. 333

Newkirk, G. A. 1961, ApJ, 133, 983

Otruba, W. 1999, in Magnetic Fields and Oscillations, ed. B. Schmieder et al., ASP Conf. Ser., 184, 314

Otruba, W., \& Pötzi, W. 2003, Hvar Obs. Bull., 27, 189

Pick, M., Malherbe, J. M., Kerdraon, A., \& Maia, D. J. F. 2005, ApJ, 631, L97 Priest, E. R., \& Forbes, T. G. 2000, Magnetic Reconnection (Cambridge: Univ. Press)

Scherrer, P. H., Bogart, R. S., Bush, R. I., et al. 1995, Sol. Phys., 162, 129

Share, G., Murphy, R. J., Smith, D. M., \& Schwartz, R. A. 2004, ApJ, 615, L169

Seehafer, N. 1978, Sol. Phys., 58, 215

Simnett, G. M. 2005, JGR, 110, A09S01

Švestka, Ž. 1984, Sol. Phys., 94, 171

Trottet, G., Chupp, E. L., Marschhäuser, H., et al. 1994, A\&A, 288, 647

Vocks, C., \& Mann, G. 2006, A\&A, 452, 331

Vršnak, B., Aurass, H., Magdalenić, J., \& Gopalswamy, N. 2001, A\&A, 377, 321 